\title{
Traduire
}

Revue française de la traduction

$223 \mid 2010$

Tribunal et théâtre, faites entrer le traducteur

\section{Réalité professionnelle de la traduction assermentée en Espagne}

Julia Lobato Patricio

Traducteur : Emmanuelle Hautbois

\section{OpenEdition}

\section{Journals}

Édition électronique

URL : http://journals.openedition.org/traduire/335

DOI : $10.4000 /$ traduire.335

ISSN : 2272-9992

\section{Éditeur}

Société française des traducteurs

\section{Édition imprimée}

Date de publication : 15 décembre 2010

Pagination : 49-65

ISSN : 0395-773X

\section{Référence électronique}

Julia Lobato Patricio, « Réalité professionnelle de la traduction assermentée en Espagne », Traduire [En ligne], 223 | 2010, mis en ligne le 10 février 2014, consulté le 17 septembre 2020. URL : http://

journals.openedition.org/traduire/335; DOI : https://doi.org/10.4000/traduire.335 


\title{
Réalité professionnelle de la traduction assermentée en Espagne
}

\author{
Julia Lobato Patricio \\ et Emmanuelle Hautbois pour la traduction/adaptation en français
}

Cet article a pour objectif de dresser le panorama actuel du métier de traducteur-interprète assermenté en Espagne. Nous nous efforcerons, à travers des explications précises et des extraits de réglementations en vigueur, de présenter les concepts clés relatifs à la traduction assermentée, les exigences relatives au format de présentation des textes, ainsi que les exigences relatives à la législation en vigueur en Espagne. Notre réflexion ainsi que nos recherches sur le métier de traducteur assermenté s'organisent comme suit :

- Différences entre la traduction juridique, la traduction assermentée et la traduction judiciaire ;

- Les compétences nécessaires au traducteur assermenté ;

- La nécessité d'effectuer des traductions assermentées ;

- Le format spécifique des traductions assermentées : cachet, signature ;

- Législation relative au traducteur assermenté en Espagne ;

- Normes de réglementation relative aux aspects méthodologiques de la traduction assermentée en Espagne ;

- Le traducteur/interprète assermenté en Espagne. Le rôle du ministère des Affaires étrangères ;

- Exemples de traductions juridiques et assermentées (anglais-espagnol) (français-espagnol).

Nous tenterons d'éclaircir certains concepts et d'expliquer les différentes facettes de la profession de traducteur assermenté. L'objectif est de présenter un travail utile et pratique tant pour le traducteur assermenté que pour les personnes désireuses de mieux appréhender la situation actuelle de la profession en Espagne. Dans la dernière partie, nous donnerons des exemples concrets de traductions assermentées accompagnées de quelques commentaires traductologiques. 


\section{Traduction assermentée : différences et similitudes avec la traduction juridique et la traduction judiciaire}

Afin de proposer une définition de la traduction assermentée, nous nous appuierons sur les concepts de traduction juridique et de traduction judiciaire. En effet, les termes de traduction juridique, traduction assermentée et traduction judiciaire renvoient à des activités apparentées mais distinctes bien que complémentaires. La traduction juridique est axée, comme son nom l'indique, sur des documents de nature juridique, c'est-à-dire à caractère légal. La traduction judiciaire est proche de la traduction juridique dans la mesure où les documents concernés revêtent souvent un aspect juridique mais ils ont pour spécificité de s'inscrire dans un processus judiciaire. II est parfois difficile de faire la distinction entre ces deux types de traduction. Nous nous efforcerons de clarifier les points qui les différencient. Enfin, la traduction assermentée, qui a pour objectif de certifier l'authenticité du document traduit, nécessite un format spécifique de présentation, que nous décrirons plus loin. La traduction assermentée est donc un type de traduction qui, tout en reprenant le même contenu, change la forme du document. Tout texte peut faire l'objet d'une traduction assermentée dont le caractère assermenté vient de sa forme et non de sa nature.

Les textes juridiques (lois, manuels de droit, etc.) n'ont pas nécessairement de valeur légale ; en revanche, les textes judiciaires ou assermentés ont une valeur légale et engagent l'auteur de la traduction, responsable devant la société d'éventuelles "erreurs de traduction ".

Afin d'éclairer notre propos selon lequel les termes traduction juridique, traduction assermentée et traduction judiciaire ne sont pas interchangeables mais complémentaires, citons le cas d'un contrat de vente, texte juridique (droit commercial) susceptible, à un moment donné, de faire l'objet d'une traduction assermentée ou d'une traduction judiciaire si le document est versé au dossier d'une procédure judiciaire.

Le tableau de la page suivante présente les caractéristiques les plus pertinentes de la traduction juridique, de la traduction assermentée et de la traduction judiciaire. 
Tableau 1 : Caractéristiques de la traduction juridique, de la traduction judiciaire et de la traduction assermentée

\begin{tabular}{|c|c|c|}
\hline Traduction juridique & Traduction judiciaire & Traduction assermentée \\
\hline $\begin{array}{l}\text { Nature du texte (oral/écrit) } \\
\text { Juridique (tous domaines } \\
\text { juridiques) }\end{array}$ & $\begin{array}{l}\text { Nature du texte (oral/écrit) } \\
\text { Tout type de traduction } \\
\text { judiciaire dans le cadre d'une } \\
\text { procédure judiciaire }\end{array}$ & $\begin{array}{l}\text { Nature du texte (oral/écrit) } \\
\text { Tout type de traduction } \\
\text { assermentée }\end{array}$ \\
\hline $\begin{array}{l}\text { Registre linguistique } \\
\text { Langage juridique, registre } \\
\text { soutenu }\end{array}$ & $\begin{array}{l}\text { Registre linguistique } \\
\text { Tout type de traduction } \\
\text { judiciaire (par ex, vulgarisation } \\
\text { s'il s'agit de la déclaration } \\
\text { d'un témoignage dans } \\
\text { un langage à niveau culturel } \\
\text { faible) }\end{array}$ & $\begin{array}{l}\text { Registre linguistique } \\
\text { Tout type de traduction } \\
\text { assermentée (normalement } \\
\text { registre soutenu) }\end{array}$ \\
\hline $\begin{array}{l}\text { Traduction accompagnée de } \\
\text { l'original? }\end{array}$ & $\begin{array}{l}\text { Traduction accompagnée de } \\
\text { l'original? }\end{array}$ & $\begin{array}{l}\text { Traduction accompagnée de } \\
\text { l'original? }\end{array}$ \\
\hline Pas nécessairement & Oui & Oui \\
\hline $\begin{array}{l}\text { Valeur légale de la traduction } \\
\text { Sans objet }\end{array}$ & $\begin{array}{l}\text { Valeur légale de la traduction } \\
\text { Oui }\end{array}$ & $\begin{array}{l}\text { Valeur légale de la traduction } \\
\text { Oui }\end{array}$ \\
\hline $\begin{array}{l}\text { Responsabilité du traducteur } \\
\text { Non }\end{array}$ & $\begin{array}{l}\text { Responsabilité du traducteur } \\
\text { Oui }\end{array}$ & $\begin{array}{l}\text { Responsabilité du traducteur } \\
\text { Oui }\end{array}$ \\
\hline $\begin{array}{l}\text { Certification } \\
\text { Non }\end{array}$ & $\begin{array}{l}\text { Certification } \\
\text { Oui }\end{array}$ & $\begin{array}{l}\text { Certification } \\
\text { Oui }\end{array}$ \\
\hline $\begin{array}{l}\text { Signature du traducteur } \\
\text { Ne doit pas apparaître }\end{array}$ & $\begin{array}{l}\text { Signature du traducteur } \\
\text { Oui }\end{array}$ & $\begin{array}{l}\text { Signature du traducteur } \\
\text { Oui }\end{array}$ \\
\hline $\begin{array}{l}\text { Formule de certification } \\
\text { Non }\end{array}$ & $\begin{array}{l}\text { Formule de certification } \\
\text { Non }\end{array}$ & $\begin{array}{l}\text { Formule de certification } \\
\text { Oui }\end{array}$ \\
\hline $\begin{array}{l}\text { Format spécifique } \\
\text { Non }\end{array}$ & $\begin{array}{l}\text { Format spécifique } \\
\text { Non, normalement le format } \\
\text { source est conservé }\end{array}$ & $\begin{array}{l}\text { Format spécifique } \\
\text { Oui (titre, formule, signature, } \\
\text { cachet, etc.) }\end{array}$ \\
\hline $\begin{array}{l}\text { Support de livraison } \\
\text { Tout support }\end{array}$ & $\begin{array}{l}\text { Support de livraison } \\
\text { Selon les instructions } \\
\text { de la procédure judiciaire } \\
\text { (normalement sur papier) }\end{array}$ & $\begin{array}{l}\text { Support de livraison } \\
\text { Uniquement papier }\end{array}$ \\
\hline
\end{tabular}




\section{Compétences du traducteur assermenté}

Nous considérons que le traducteur spécialisé en traduction juridique (ce qui pourrait également être applicable à la traduction judiciaire et à la traduction assermentée) doit présenter des compétences spécifiques, comme le souligne Carmen Mata Pastor, professeure à l'Université de Málaga, dans sa thèse de doctorat: Acercamiento a la traducción de textos de naturaleza jurídica italiano-español. Un caso práctico: El arbitraje. [Approche relative à la traduction de textes de nature juridique de l'italien vers l'espagnol. Un cas pratique : l'arbitrage.]

Compétences linguistiques :

- une excellente connaissance des deux langues concernées ;

- une certaine connaissance du latin et des latinismes utilisés dans ses langues de travail ;

- une connaissance approfondie de la rhétorique propre au langage juridique dans les deux langues ;

- la capacité à reconnaître, interpréter et reproduire les archaïsmes.

Compétences textuelles :

- la capacité à identifier la typologie textuelle dans le domaine légal correspondant et à traiter la documentation textuelle.

Compétences extralinguistiques :

- des connaissances de base du système juridique des pays concernés et la connaissance des thèmes traités ;

- la capacité à retranscrire l'acte de communication du texte source (TS).

Compétences psychophysiologiques :

- une bonne mémoire à court terme et à moyen terme (interprétation) ;

- la capacité de se rendre compte des éventuelles pressions exercées, la rigueur (cachets, tampons, etc.) et la conscience de la portée de ses fonctions ;

- la facilité de prise de décision (consacrer des termes, établir des équivalences, intervenir sur les mémoires de traduction, etc.) ;

- une certaine "psychologie".

Compétences d'adaptation :

- une certaine souplesse vis-à-vis d'une autorité supérieure (interprétation devant un juge, un notaire, interventions d'office), associée à une certaine confiance en soi et à une indépendance face à un quelconque type de pression. 
- des compétences professionnelles et relationnelles : indépendance, sincérité et transparence face aux clients ;

Compétences stratégiques :

- la capacité à évaluer la portée des décisions dans la rédaction d'un document d'ordre juridique ou amené à devenir un instrument juridique.

Nous devons ajouter à ces compétences un dernier élément que nous considérons important, non seulement pour l'exercice professionnel de la traduction juridique mais également pour tout type de traduction : les compétences critiques. Le traducteur doit avoir un regard critique sur ses sources de documentation dont il doit être capable d'évaluer la fiabilité. Outre cet esprit critique, l'existence d'un esprit d'autocritique est fondamentale à toute traduction, c'est-àdire que le traducteur doit être capable de prendre un recul suffisant par rapport à sa propre traduction pour obtenir une qualité optimale.

La question de la formation est aussi un point souvent débattu : les compétences du traducteur sont-elles innées ou doit-il, au contraire, les acquérir par le biais d'une formation en traduction ou en interprétation. II est entendu que certains individus ont des prédispositions pour telle ou telle discipline ou sont plus ou moins capables d'assumer certaines tâches. Nous considérons toutefois que l'exercice de la traduction professionnelle dans le secteur juridique nécessite une formation spécialisée, même si les aptitudes sociales de certaines personnes leur permettront parfois d'exceller dans leur profession.

\section{Quelle est l'utilité des traductions assermentées ?}

La réalisation de ce type de traduction est déterminée par les exigences des autorités compétentes (ministères, tribunaux, institutions scolaires) qui souhaitent un exemplaire fidèle et certifié d'un document original déterminé, par la nécessité de consigner un exemplaire certifié d'un document original ou encore par la nécessité de transcrire, dans la langue cible, les informations d'un texte source. Les traductions assermentées dans les règles de l'art deviennent publiques une fois apposé le cachet de certification ou la signature de l'interprète ou du traducteur assermenté.

\section{Le format de la traduction assermentée}

Les traductions assermentées exigent un format spécifique de présentation qui respecte les points suivants :

a) Titre et entête : la traduction assermentée doit débuter par un titre ou un entête dans lequel apparaît : "Traduction assermentée de [type de document] ". 
b) Traduction à proprement parler : la traduction est insérée après le titre ou l'entête.

c) Formule de certification : texte bref par lequel le traducteur assermenté certifie la fidélité et la qualité de la traduction par rapport au texte source.

Dans le décret royal 79/1996 du 26 janvier, publié le 23 février 1996, le ministère des Affaires étrangères indique la formule de certification suivante :

Don / Doña (nombre y apellidos), Intérprete Jurado de (idioma), certifica que la que antecede es traducción fiel y completa al (lengua de destino) de un documento redactado en (lengua de origen). En (lugar), a (fecha).

\section{FIRMA}

[Monsieur/Madame de (langue), atteste que le texte ci-dessus constitue une traduction fidèle et complète vers source).

À (lieu), le (langue cible) d'un document rédigé en (langue

\section{SIGNATURE}

d) Paraphe : initiales apposées sur chacune des pages de la traduction, à l'exception de la dernière page qui comporte la signature.

e) Signature, date et cachet du traducteur assermenté : la signature complète et la date sont insérées à la fin du document ; cependant, le cachet doit être inséré sur chaque page de la traduction, près du paraphe, ainsi que sur la dernière page.

f) Annexe avec la copie de l'original : une traduction assermentée doit être accompagnée d'une copie du document original en annexe puisqu'elle présente un caractère officiel et que, dans de nombreux cas, celui-ci est utilisé pour authentifier les informations traduites.

En cas de nécessité, la traduction assermentée peut contenir, au-delà des notes explicatives en pied de page, une série d'annotations ou d'explications que le traducteur considère appropriées, entre crochets [...].

Dans l'illustration suivante, nous présentons un exemple de traduction assermentée sur laquelle tous les éléments caractéristiques précédemment décrits sont indiqués. Dans ce cas, la traduction a été réalisée sur un formulaire officiel. Bien qu'il ne soit pas obligatoire de présenter une traduction assermentée utilisant ce type de formulaire, certaines administrations publiques se montrent plus coopérantes lorsqu'elles reçoivent des documents imprimés sur papier officiel ; elles considèrent, à tort, que ce type de support confère un caractère plus 
officiel à la traduction. Une traduction assermentée n'est pas un document officiel du fait du type de papier sur lequel elle est imprimée mais du fait de la signature et du cachet que le traducteur-interprète assermenté appose, sous autorisation du ministère des Affaires étrangères et de la coopération.

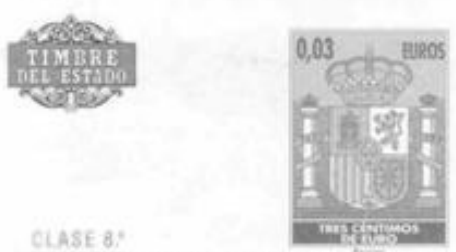

TRADUCCION JURADA DE INGLÉS A ESPAÑOL. DE UNA APOSTIL.I.A DE LA HAYA

\section{ESTADO DE WISCONSIN}

Titre

Secretaria de Estado

APOSTIL.L.A.

(Coavention de La Haye du 5 Octobre 1961)

1. Pais: Estados Unidos de América-

2. El presente documento puiblico ha sido firmado pot: Cathy Willsquette.

3. Quien actúa en calidad de: responsable del registro de documentos públicos del condado de Brown, Wisconsin-

4. y está revestido del sello de dicha secretaria-

CERTHFICADO para España-

\section{Corps de texte}

5. En Madison, Wisconsin- 6. el 19 de agosto de 2009 -

7. Por el Sectetario de Fstado de Wisconsin-

8. Bajo el número: 183557 , -

9. Selloc [Aparece un sello dorado en relieve con una inscripción que rezx: "Great seal of the State of Wisconsin" Gran sello del Estado de Wisconsin]-

10. Firma [Rúbrica ilegible]-DOUGl.AS LA FOLLETTE-Secretario de Estado-

Esta apostilla sólo certifica la autoridad de la persona citada en el apartado número 2. No implica que los contenidos del documento adjunto estén correctos ni que el Secretario de Estado esté de acuerdo con el contenido.

En la parte superior detecha aparece un sello en color arul que reza: gran sello del estado de Wisconsin. CFR'THFICADO.

Doña Julia Lobato Patricio, intérprete jurado de inglés autorizada por el Ministerio de Asuntos Exteriores, certifiea que I Formule de certification
traducción fiel y completa al español de un documento re

Y para que surta los efectos oportunos donde y cuando proceda la firma y extiende, a petición del interesado/a en Málaga, a 24 de agosto de 2009.

Esta traducción impresa en papel timbrado del Estado con la numeración $0 . J 1751915$, consta de 1 página firmada y sellada.

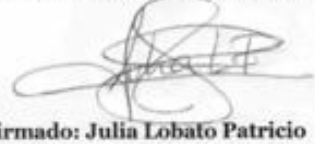

Signature

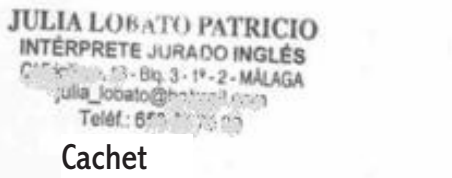




\section{Voies d'accès au titre de traducteur-interprète assermenté en Espagne}

Le traducteur-interprète assermenté est une personne reconnue et habilitée par le ministère des Affaires étrangères à effectuer des traductions officielles depuis et vers l'espagnol, de tout type de document rédigé dans la langue étrangère pour lequel il est assermenté.

En Espagne, jusqu'à maintenant, il existe deux voies d'accès au titre de traducteur-interprète assermenté :

a) Se soumettre à un examen organisé par le ministère des Affaires étrangères, conformément au décret du 8 février 1996, lequel précise le contenu des épreuves visant à l'obtention du titre d'interprète-traducteur assermenté, BOE [Bulletin Officiel de l'État], du 23 février 1996.

Les examens des traducteurs-interprètes assermentés comportent quatre épreuves, chacune ayant un caractère éliminatoire :

- Traduction vers l'espagnol, sans dictionnaire, d'un texte littéraire ou d'un article de journal ;

- Traduction de l'espagnol vers une autre langue, sans dictionnaire, d'un texte littéraire ou d'un article de journal ;

- Traduction vers l'espagnol, avec dictionnaire, d'un texte juridique ou économique ;

- Épreuve orale : le candidat doit prouver devant le Tribunal sa capacité de compréhension et d'expression orale dans la langue pour laquelle il souhaite être assermenté.

À cet effet, le candidat doit résumer oralement un texte écrit qui lui est remis par le Tribunal ; il doit également commenter ce texte en répondant aux questions qui lui sont posées.

b) Être titulaire d'un master (1) en traduction-interprétation ou d'un diplôme étranger équivalent attestant d'" une préparation spécifique en traduction juridique et économique et en interprétation dans la langue ou dans les langues pour lesquelles le traducteur souhaite être assermenté "; cette préparation étant constituée d'un minimum de 24 crédits en traduction juridique ou économique et de 16 crédits en interprétation.

Nous avons utilisé l'expression " jusqu'à maintenant " puisque récemment, le règlement du Bureau de l'Interprétation des langues du ministère des Affaires étrangères a été modifié par décret royal(2). Le Bureau de l'Interprétation des langues (OIL) est le principal organe public traitant de la traduction et de l'interprétation.

(1) Décret AEX/1971/2002, du 12 juillet, dans lequel sont établis les exigences et le processus d'obtention du titre d'interprète assermenté pour les titulaires d'un master en traduction et interprétation, joint en annexe.

(2) Décret royal 2002/2009, du 23 décembre, dans lequel est modifié le Règlement du Bureau de l'Interprétation des langues du ministère des Affaires étrangères, approuvé par le Décret royal 2555/1977, du 27 août. 


\section{La législation relative au titre de traducteur-interprète assermenté en Espagne}

Nous présentons ci-après la législation espagnole réglementant le statut de traducteur-interprète assermenté, dénomination modifiée par le dernier décret royal. Auparavant la dénomination officielle était " interprète assermenté " mais la législation admettait que l'interprète assermenté puisse exécuter des traductions orales comme écrites.

Les textes officiels sont les suivants:

- Décret royal 2555/1977, du 27 août par lequel est approuvé le règlement relatif au Bureau de l'Interprétation des langues du ministère des Affaires étrangères ;

- Décret royal 79/1996 du 26 janvier, publié le 23 février 1996, qui modifie plusieurs articles du décret royal 2555/1977 ;

- Décret du 8 février 1996 dans lequel sont émises des normes relatives aux examens pour l'obtention du titre des interprètes assermentés, BOE du 23 février 1996 ;

- Décret AEX/1971/2002, du 12 juillet, dans lequel sont établies les exigences et le processus d'obtention du titre d'interprète assermenté pour les diplômés de Master de traduction et interprétation ;

- Décret royal 2002/2009, du 23 décembre, dans lequel est modifié le Règlement du Bureau de l'Interprétation des langues du ministère des Affaires étrangères, approuvé par le décret royal 2555/1977, du 27 août.

Le décret royal 2002/2009 du 23 décembre pose quelques modifications importantes de la profession de traducteur assermenté.

Tout d'abord, l'interprète assermenté sera désormais nommé " traducteur-interprète assermenté " puisqu'il ne travaille pas uniquement sur des textes oraux (interprétations) mais également sur des textes écrits (traductions).

Une autre modification proposée par ce décret royal est le moyen d'accès au titre de traducteur-interprète assermenté. Ce moyen d'accès est maintenant limité au passage de l'examen annuel du ministère des Affaires étrangères et de la coopération.

Dernière modification importante : il ne sera plus nécessaire de communiquer ses tarifs tous les ans au Bureau de l'Interprétation des langues afin de figurer dans l'annuaire des traducteurs-interprètes assermentés. 


\section{Normes relatives aux aspects méthodologiques de la traduction assermentée}

II n'existe à ce jour aucune norme obligatoire réglementant les traductions assermentées en termes de méthodologie. L'Association professionnelle espagnole des traducteurs et interprètes (A.P.E.T.I.) a toutefois publié en 1992 un ensemble de normes transitoires relatives à la traduction assermentée, ce qui constituait la première tentative de normalisation dans ce domaine. Bien que ces normes n'aient pas été appliquées, nous considérons qu'elles sont complètes et qu'elles recouvrent les aspects fondamentaux à prendre en compte lors de la réalisation d'une traduction assermentée. Elles constituent des éléments d'orientation pouvant guider les professionnels et les étudiants.

D'une part, Luis Márquez Villegas présente une synthèse de ces normes dans l'ouvrage Introducción a la traducción jurídica y jurada (inglés-español) (3) et d'autre part, Miguel Duro Moreno propose une normalisation stylistique de la traduction assermentée dans son article "La traducción jurada : propuesta de normalización estilística " [La traduction assermentée : proposition de normalisation stylistique] dans l'ouvrage La traducción e interpretación jurídicas en la UE. Retos para la Europa de los ciudadanos [La traduction et l'interprétation juridiques dans l'UE. Défis pour l'Europe des citoyens].

\section{Exemples de traductions assermentées}

\subsection{Original $n^{\circ} 1$ :}

Objet de la traduction : il nous est demandé de réaliser une traduction assermentée de l'attestation ci-dessous. Notre client a besoin d'une traduction officielle qu'il doit présenter à une université espagnole pour valider ses stages dans un établissement d'enseignement secondaire au Royaume-Uni en vue de l'obtention du Certificado de Adaptación Pedagógica (CAP) [Certificat d'aptitude pédagogique].

(3) p. 99-113 


\section{Traduction de l'original $n^{\circ} 1$ :}

\section{TRADUCCIÓN JURADA DE UN NOMBRAMIENTO DE AUXILIAR DE CONVERSACIÓN CENTROS DE EDUCACIÓN PRIMARIA Y SECUNDARIA}

"[En el documento original aparecen ciertas partes ya traducidas al español].-.

[En la parte superior izquierda aparece el logotipo del British Council]. Nombramiento de Auxiliares de ConversaciÛn en Escuelas y Colegios.Certificado del Servicio.-

Por el presente certificado se hace constar que XXXXXX XXXXX trabajó como Auxiliar de Conversación, de acuerdo con el plan oficial llevado a cabo por el British Council en nombre de los departamentos de Educación de Reino Unido, en los dos centros de educación secundaria y de carácter público siguientes: THE ROYAL GRAMMAR SCHOOL, High Wycombe, Amersham Road, High Wycombe, HP13 6QT y SIR WILLIAM RAMSAY SCH00L durante el año académico 2004-2005 y desde el 1 de octubre al 31 de mayo de ese año. Asimismo, XXXXX trabajó doce horas a la semana en dichos centros impartiendo clases de Español [como lengua extranjera] a alumnos pertenecientes a los niveles que abarcan entre Year 9 [tercer nivel de los siete que conforman la Educación Secundaria en Reino Unido y que agrupa alumnos de entre 13 y 14 años] y Year 13 [nivel superior de la Educación Secundaria no obligatoria en Reino Unido cuyos alumnos comprenden edades entre 17 y 18 años].-........ Joan Hoggan, Jefe de la Sección de Auxiliares de Conversación [Rúbrica ilegible].--

Departamento de Educación y Empleo.-.

Fecha de expedición: 22 de febrero de 2006.-

Departamento de Educación de Gales.-

Certificado del Servicio de Auxiliar de Conversación de Español.-

El British Council es la organización internacional de Reino Unido cometida a las relaciones educativas y culturales.-..

El British Council está registrado en Inglaterra como institución benéfica. "

Dña. XXXXX XXXXXX, Intérprete Jurado de inglés, certifica que la que antecede es traducción fiel y completa al español de un documento redactado en inglés.

La traducción consta de una página numerada, firmada y sellada.

En lugar, a día de mes de año.

SELLO y FIRMA

Fdo.: XXXXXX XXXXXXXXX 


\section{Commentaires sur la traduction :}

Nous indiquerons dans notre traduction, entre crochets, que le document original est sous format partiellement bilingue.

Les lignes ont été complétées par des traits de façon à éviter toute modification de la traduction.

Nous avons retiré le nom de la personne sur le document original et sur la traduction, de façon à préserver sa vie privée. Les noms propres, par ex. le nom du Centre, et les adresses ne se traduisent pas. Des précisions ont été ajoutées sur le système scolaire britannique. Par souci de clarté, nous avons choisi de placer entre crochets le niveau d'études correspondant dans le système scolaire espagnol et l'âge moyen des élèves qui atteignent ce niveau.

Au terme de la traduction, la formule de certification établie par le ministère des Affaires étrangères et de la coopération (MAEC) doit être insérée. Bien que le MAEC ne fasse aucune référence à cet élément, il est recommandé d'indiquer le nombre de pages que compte la traduction. Enfin, le cachet ainsi que la signature du traducteur-interprète assermenté auprès du MAEC sont apposés.

\subsection{Original $n^{\circ} 2$ :}

\section{Objet de la traduction :}

Notre client, de nationalité britannique, souhaite se marier en Espagne. Afin de remplir les formalités administratives requises, il souhaite une traduction assermentée de son certificat de naissance avec l'apostille correspondante de La Haye. Selon la Convention de La Haye du 5 octobre 1961, l'exigence de la légalisation diplomatique ou consulaire des actes publics étrangers a été supprimée.

Nous présentons donc ci-après le document original, duquel nous avons supprimé les données personnelles à des fins de confidentialité, suivi de la traduction assermentée. 


\section{CERTIFIED COPY}

Pursuant to the Births and
BU 769031

\section{OF AN ENTRY}

Deaths Registration Act 1953

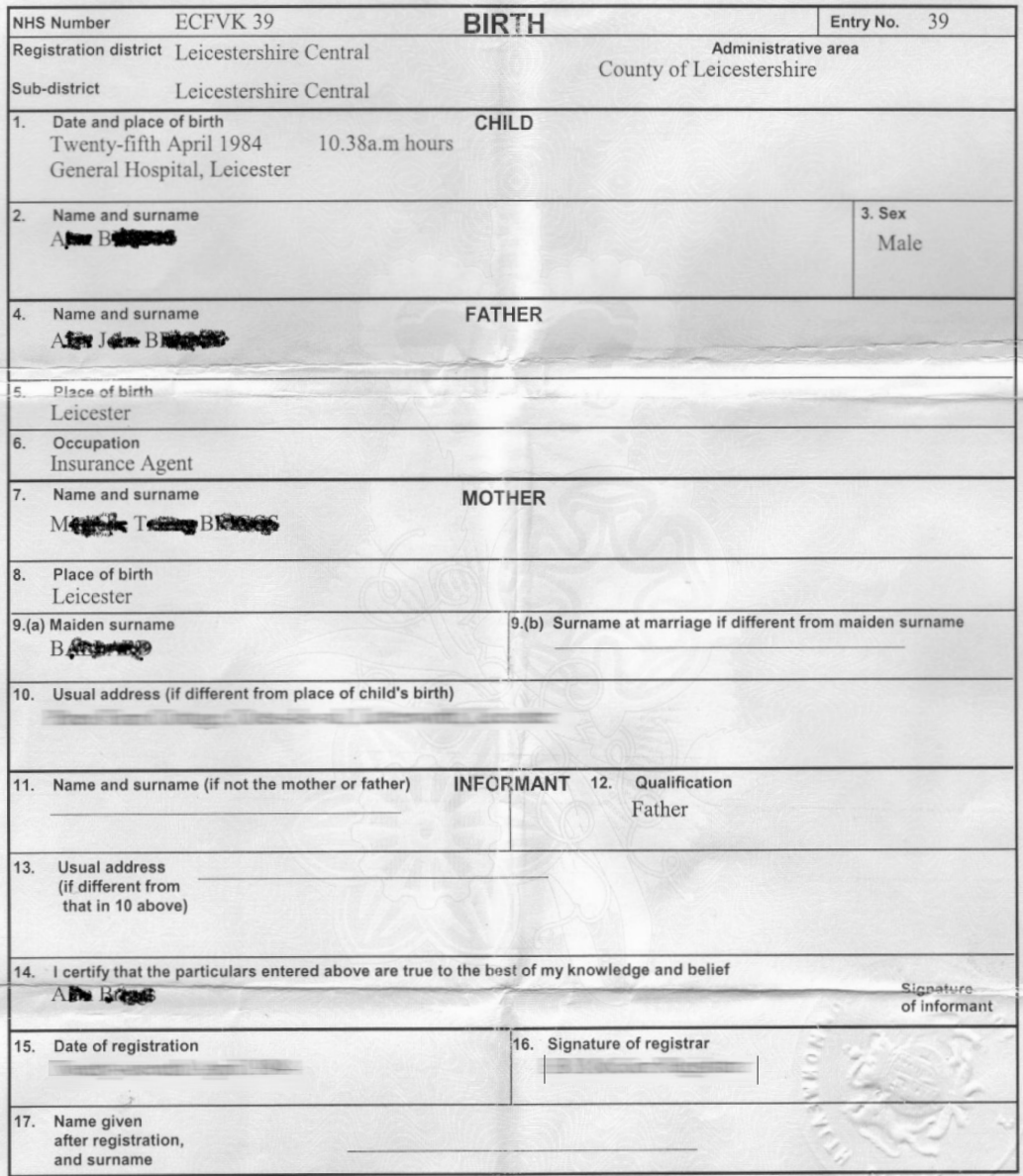

Certified to be a true copy of an entry in a register in my custody.
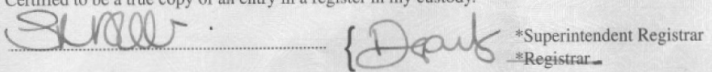

*Strike out whichever does not apply

CAUTION: THERE ARE OFFENCES RELATING TO FALSIFYING OR ALTERING A CERTIFICATE AND USING

OR POSSESSING A FALSE CERTIFICATE. 'CROWN COPYRIGHT

WARNING: A CERTIFICATE IS NOT EVIDENCE OF IDENTITY. 


\section{Traduction de l'original $\mathrm{n}^{\circ} 2$ :}

TRADUCCIÓN JURADA AL ESPAÑOL DE UN CERTIFICADO DE NACIMIENTO REDACTADO EN INGLÉS CERTIFICADO DE NACIMIENTO.- Conforme a la Ley de Inscripción de nacimientos y defunciones de 1953 [del Reino Unido].- [En la parte superior derecha aparece: BU 769031.-

[En la parte superior central aparece un escudo del Reino Unido de Gran Bretaña e Irlanda del Norte].-

Número del NHS [Servicio Nacional (Británico) de Salud]: ECFVK 39.- NACIMIENTO. Entrada No. 39.Distrito de inscripción: Leicestershire Central.- Área administrativa: Condado de Leicestershire.Sub-distrito: Leicestershire Central.--

NIÑO/A.-

1. Lugar y fecha de nacimiento: General Hospital [Hospital General], Leicester, veinticinco de abril de 1984 a las $10: 38$ horas.-

2. Nombre y apellido: A... B...- 3. Sexo: Hombre.-

PADRE.-

4. Nombre y apellido: A... J... B...- 5. Lugar de nacimiento: Leicester.- 6. Profesión: Agente de seguros.-

MADRE.-

7. Nombre y apellido: M... T... B...-8. Lugar de nacimiento: Leicester.-9.(a) Nombre de soltera: B...9.(b) Apellido en el momento de contraer matrimonio en caso de no coincidir con el de soltera: [espacio tachado].- 10. Domicilio habitual (en caso de no coincidir con el del lugar de nacimiento del niño/a): P..., C..., L..., Leicester.-

DECLARANTE.-.

11. Nombre y apellido (en caso de ser una persona distinta al padre o la madre): [espacio en blanco]. 12. En calidad de: Padre.- 13. Domicilio habitual (en caso de ser distinto al indicado en el apartado 10): [espacio tachado].-.

14. Certifico que todos los datos consignados en el presente documento son veraces a mi leal saber y entender.- Firma del declarante: A... B...-

15. Fecha de inscripción: veintisiete de abril de 1984.- 16. Firma del registrador: Registrador B.R. M...-

17. Nombre tras la inscripción en el registro y apellido [espacio tachado].-

[Aparece una IInea en la parte inferior central].-

Certifico que es copia fiel de una entrada inscrita en un registro que se encuentra bajo mi tutela.- [Nombre ilegible escrito a mano] Responsable Adjunto ["adjunto" escrito a mano] del registro.- Fecha: 22.12.05 [escrita a mano].-

AVISO: EXISTEN DELITOS RELACIONADOS CON LA FALSIFICACIÓN O ALTERACIÓN DE CERTIFICADOS Y CON EL USO Y POSESIÓN DE CERTIFICADOS FALSOS. @ CROWN COPYRIGHT [derechos de autor de la Corona].-

ADVERTENCIA: UN CERTIFICADO NO ES UNA PRUEBA DE IDENTIDAD.-

[El documento original posee una marca de agua con forma de flor en tonos rosas]"

Dña. XXXXX XXXXXX, Intérprete Jurado de inglés, certifica que la que antecede es traducción fiel y completa al español de un documento redactado en ingles.

La traducción consta de una página numerada, firmada y sellada.

En lugar, a día de mes de año.

SELLO y FIRMA

Fdo.: XXXXXXXXXXXXXXX 


\section{Pistes de recherches sur la traduction assermentée}

Lorsqu'un étudiant de master décide d'effectuer des recherches pour son mémoire ou sa thèse, il est bien souvent désorienté par la quantité de pistes de recherches existantes dans le type de traduction choisi. Nous proposons ci-après quelques pistes de recherches potentielles dans le domaine de la traduction assermentée, pistes qui ont pour objectif d'orienter l'étudiant. Bien que certaines de ces propositions aient déjà été traitées par certains chercheurs, elles pourront être approfondies ou traitées sous un angle différent. La traduction assermentée offre une grande diversité de champs de recherche parmi lesquels nous nous contenterons de citer :

- Didactique de la traduction juridique et de la traduction assermentée.

- Méthodologie de la traduction juridique et de la traduction assermentée.

- La traduction juridique, la traduction assermentée et les nouvelles technologies.

- La traduction assermentée des documents à caractère légal (successions, contrats, actes notariés).

jlobato@upo.es ; e_hautbois@yahoo.fr

Julia LOBATO PATRICIO est traductrice assermentée indépendante anglais <>espagnol, membre d'ASETRAD, et professeure de traduction spécialisée (français>espagnol) à l'Université Pablo de Olavide de Séville, en Espagne depuis 2009. Elle a aussi enseigné à l'Université de Cordoue, en Espagne. Elle a soutenu sa thèse de doctorat à l'Université de Málaga en 2008, sur l'éthique de la traduction juridique. Celle-ci s'intitule Aspectos deontológicos y profesionales de la traducción jurídica, jurada y judicial [Aspects déontologiques et professionnels de la traduction juridique, assermentée et judiciaire].

Emmanuelle HAUTBOIS est traductrice indépendante (anglais/espagnol>français), membre de la SFT. Elle est aussi chargée d'enseignement en traduction spécialisée (espagnol>français) au CFTTR Université de Rennes 2 - Haute Bretagne, en France. 


\section{Bibliographie}

ALCARAZ VARO, Enrique (1994) : El inglés jurídico: textos y documentos, Ariel, Barcelone.

ALCARAZ VARO, Enrique et HUGHES, Brian. (2002) : El español jurídico, Ariel Derecho, Barcelone.

ALCARAZ VARO, Enrique (2007) : Diccionario de términos jurídicos (inglés-español, españolinglés), 10e édition. Ariel Derecho, Barcelone.

BORJA ALBI, Anabel (2000) : El texto jurídico inglés y su traducción al español, Ariel Lenguas Modernas, Barcelone.

DURO MORENO, Miguel «La traducción jurada : propuesta de normalización estilística (inglés-español / español-inglés) (francés-español/español-francés)" in E. Ortega Arjonilla (dir.) (2008) : La traducción e interpretación jurídicas en la UE. Retos para la Europa de los ciudadanos. Grenade, E. Comares, collection Interlingua.

HURTADO ALBIR, Amparo (2004) : Traducción y traductología. Introducción a la traductología, 2e édition, Cátedra Lingüística, Madrid.

KELLY, Dorothy (dir.) (2000), La traducción y la interpretación en España hoy: perspectivas profesionales. Editorial Comares, Grenade.

LOBATO PATRICIO, Julia (2008). Aspectos deontológicos y profesionales de la traducción jurídica, jurada y judicial. Thèse de doctorat. Servicio de publicaciones de la Universidad de Málaga.

MATA PASTOR, Carmen Acercamiento a la traducción de textos de naturaleza jurídica italianoespañol. Un caso práctico: El arbitraje : Thèse de doctorat. Universidad de Málaga.

MAYORAL ASENSIO, Roberto (2003). " Investigación en traducción jurada ». Panorama actual de la investigación en traducción e interpretación (coord.) García Peinado, M. A. \& Ortega Arjonilla, E. ISBN 84-96101-11-X, pp. 457-467.

ORTEGA ARJONILLA, Emilio (2005) : " La traducción jurídica, jurada y judicial: aspectos teóricos, metodológicos y profesionales. "En: Borja Albí, A. y Monzó Nebot, E. Traducción y mediación en las relaciones jurídicas internacionales. Universitat Jaume I, Castellón.

ORTEGA ARJONILLA, Emilio et alii (2005) : Traducción jurídica, jurada y judicial (francés-español). Aspectos teóricos, metodológicos y profesionales. Editorial Comares, colección Interlingua $n^{\circ} 1$, Grenade (3e édition corrigée et améliorée).

ORTEGA ARJONILLA, Emilio (dir.) (2008) : La traducción e interpretación jurídicas en la UE. Retos para la Europa de los ciudadanos, Grenade, Éd. Comares, collection Interlingua. 
SAN GINÉS AGUILAR, P. \& ORTEGA ARJONILLA, Emilio (1997): Introducción a la traducción jurídica y jurada (inglés-español). Éd. Comares, Grenade, 2e édition.

\section{Textes officiels}

Convention de La Haye du 05 octobre 1961 supprimant l'exigence de la légalisation des actes publics étrangers.

Décret AEX/1971/2002, du 12 juillet, par lequel sont établis les exigences et le processus relatifs à l'obtention du titre d'interprète assermenté pour les diplômés de Master en traduction et en interprétation.

Décret du 8 février 1996, par lequel sont édictées les normes relatives aux examens pour obtenir le titre d'interprète assermenté, BOE, 23 février 1996.

Décret royal 2002/2009, du 23 décembre, portant modification à la Réglementation du Bureau de l'Interprétation des langues du ministère des Affaires étrangères, approuvé par Décret royal 2555/1977, du 27 août.

Décret royal 2555/1977, du 27 août, par lequel est approuvée la Réglementation du Bureau de l'Interprétation des langues du ministère des Affaires étrangères.

Décret royal 79/1996 du 26 janvier, publié le 23 février 1996, qui modifie plusieurs articles du DR 2555/1977. 